\title{
SITUACIÓN DE LOS CENTROS DE DOCUMENTACIÓN EN LOS MEDIOS ESCRITOS ANDALUCES
}

\author{
Situation of documentation services in the \\ Andalusian written media
}

Natalia Meléndez-Malavé y Rocío Hirschfeld-Suárez

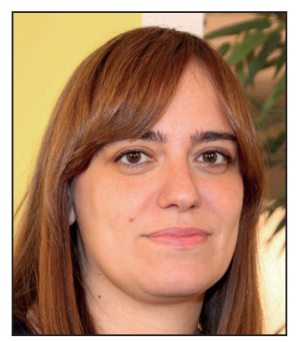

Natalia Meléndez-Malavé, doctora en periodismo (2005) por la Universidad de Málaga (UMA), es profesora en el Departamento de Periodismo de la Facultad de Ciencias de la Comunicación de la UMA, donde imparte la asignatura Documentación informativa en el grado de periodismo. Es integrante de varios grupos de investigación nacionales y de proyectos de innovación educativa en la enseñanza universitaria. Ha publicado diversas aportaciones en obras, actas de congresos y artículos en revistas especializadas con material hemerográfico como fuente para la investigación, así como sobre la adaptación de metodologías didácticas innovadoras para la impartición de la asignatura Documentación informativa. http://orcid.org/0000-0003-0835-3997

Universidad de Málaga, Facultad de Ciencias de la Comunicación, Departamento de Periodismo Campus Teatinos, s/n, 29071 Málaga, España natalia@uma.es

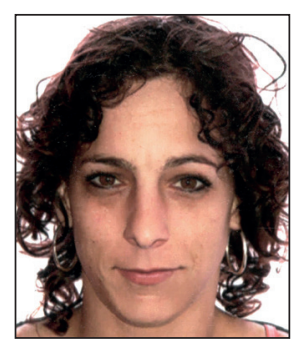

Rocío Hirschfeld-Suárez es estudiante de periodismo en la Facultad de Ciencias de la Comunicación de la Universidad de Málaga, en la cual además ha realizado diversos cursos de formación complementaria en la rama del periodismo político o referentes a temas como el lenguaje de los medios en la formación de la imagen femenina. Efectuó sus prácticas laborales en la cadena local Onda Azul Málaga RTV, realizando tareas de redacción, montaje y locución.

http://orcid.org/0000-0003-0602-1761

rociohirschfeld@hotmail.com

\section{Resumen}

Este trabajo analiza la situación actual de los departamentos de documentación en 14 de las principales cabeceras locales andaluzas. El objetivo es actualizar los datos de un estudio similar titulado "Situación de los servicios de documentación de la prensa diaria de Andalucía" (Aquesolo-Vegas, 1996) que realizaba una radiografía de estos departamentos en siete diarios. Este trabajo recoge, adapta y amplía la metodología de aquella investigación con el fin de conocer cómo ha evolucionado desde entonces el panorama de estos centros en los periódicos andaluces más representativos. Con ese fin se ha realizado un estudio de campo basado en cuestionarios, enviados a 16 diarios andaluces, dos por provincia, de los cuales 14 fueron contestados. Se analizan aspectos como las tareas, el personal, las instalaciones y el equipamiento. Los resultados reflejan, a la par que una reconversión tecnológica, la reducción e incluso la desaparición de estos servicios, en una etapa lastrada por la crisis económica y caracterizada por la búsqueda autónoma de documentos por parte de los redactores.

\section{Palabras clave}

Servicios de documentación; Documentación periodística; Periodismo; Prensa; Diarios; Medios; Andalucía; Crisis de la prensa escrita.

\footnotetext{
Abstract

This paper analyzes the situation of the archive departments in 14 of the Andalusian local newspapers. The aim is to update a similar study entitled "Situation of archive services in the daily press of Andalusia" (Aquesolo-Vegas, 1996) in which an analysis of these departments was performed for seven newspapers. This work studies, adapts, and extends the methodology of the above cited study to know how the situation has evolved since 1996. To that end, this field study is based on questionnaires sent to 16 Andalusian newspapers, two per province; 14 of which were completed. Aspects such as tasks, staff, facilities, and equipment are analyzed. The results show a technological restructuring, and also a reduction, or even the disappearance, of these services, due to the economic downturn. Therefore, journalists must now search for documents by themselves.
} 


\section{Keywords}

Document management; Archive department; News librarianship; Journalism; Press; Newspapers; Media; Andalusia; Crisis of the press.

Meléndez-Malavé, Natalia; Hirschfeld-Suárez, Rocío (2016). "Situación de los centros de documentación en los medios escritos andaluces". El profesional de la información, v. 25, n. 4, pp. 606-615.

http://dx.doi.org/10.3145/epi.2016.jul.10

\section{Introducción}

La documentación forma parte de cualquier proceso de conocimiento $y$, por ende, de todo proceso informativo (Galdón-López, 2002, p. 67). La profesión periodística, debido a la naturaleza de su labor, es una de las que más necesita "documentarse para completar y verificar las noticias recientes que se van a publicar" (Torres-Rodríguez, 2012 , p. 29). Es decir, una información fundamentada que permita un conocimiento crítico del pasado, y que ayude al público a comprender las causas y consecuencias de los hechos que acontecen.

Con ese fin, desde sus orígenes entre finales del siglo XIX y principios del $\mathrm{XX}$, se fueron configurando en los medios de comunicación los centros de documentación como el perfecto intermediario entre la información y el periodista al "ser los recursos más capacitados para buscar y obtener información" (Rubio-Lacoba, 2005, p. 156). Paralelamente, se va desarrollando la Documentación Informativa como disciplina, que hoy conforma una materia indiscutible en los planes de estudio, entre otras, de las titulaciones de Periodismo.

En España, hasta mediados de los 70, la mayoría de los medios de comunicación escritos españoles "carecían incluso de terminales para el procesamiento de textos. La automatización de los periódicos se centró en la automatización de los procesos de composición e impresión". Aunque, cabe reseñar que, en la década de los 80 , ya existían proyectos serios para la informatización de archivos (MoreiroGonzález, 2000, p. 60). Como afirman Marcos-Recio y Edo (2015, p. 391):

"La transformación de los medios de comunicación se inició con la implantación de los ordenadores en las redacciones. Allá por los años ochenta del siglo pasado se rompió una estructura de hacer y servir la información que se conservaba desde los tiempos de la imprenta".

Así, a partir de la década de los 90, el panorama de la actividad documental informativa española experimenta grandes cambios. No sólo porque en ese periodo comienzan a proliferar los manuales sobre Documentación Informativa (González-Quesada, 2003, p. 77), sino, además, a causa de que la ineludible informatización, en mayor o menor grado, de los departamentos de documentación se convierte en un hecho. La digitalización de los archivos traerá grandes mejoras para los documentalistas, pero pese a esta circunstancia, es un número muy elevado el de periodistas que optan por buscar la información que requieren para la elaboración de sus trabajos en el ciberespacio antes que en los departamentos de documentación. En 2009, un es- tudio de Micó-Sanz; Masip y García-Avilés (2009, p. 287) en el que fueron entrevistados más de 70 profesionales de la información y se les realizó una encuesta a los responsables de los centros de documentación de las televisiones y los periódicos más relevantes del país, en ese momento, reflejaba que el empleo de buscadores como Google y distintas hemerotecas accesibles por la red se ha extendido entre los profesionales de la información por encima del uso de la base de datos del propio medio, colocándose como primeras fuentes de consulta.

De este modo, llegamos al contexto actual de descenso del peso de los servicios de documentación en los medios, debido en parte a que los usuarios han ido adquiriendo mayores competencias en la búsqueda autónoma de documentación, lo que no debe ser obstáculo, sino, al contrario, un impulso a los profesionales para contrarrestar el escenario que describen algunos autores:

“Esta situación de autonomía en la búsqueda y recuperación de información en sistemas integrados puede dar lugar a un menor contacto y diálogo entre usuarios y documentalistas, lo que conlleva un distanciamiento entre el análisis que realizan los documentalistas y las necesidades de información de los usuarios" (Estrada-Nora González; Patallo-Fernández; Pastor-Blanco, 2009, p. 331).

Por su parte, Orero y Cebrián-Enrique concluyen que

"expertos y documentalistas consideran que las fuentes digitales han mejorado la calidad del servicio de las unidades de información por el crecimiento exponencial de los recursos y la rapidez en la respuesta de las consultas. Sin embargo, se confirma que el uso de internet en las redacciones ha provocado también una pérdida de control de los documentalistas sobre las búsquedas de los usuarios" (Orero; Cebrián-Enrique, 2014, p. 615).

Paralelamente, como explica Javier Guallar:

"la crisis en la que están inmersos los medios de comunicación en general y en especial la prensa escrita en la actualidad, está diezmando el personal de las redacciones, afectando de lleno a documentalistas y a centros de documentación" (Guallar, 2011b, p. 54).

Así, la situación económica de los últimos años -sumada al surgimiento de nuevas plataformas- ha afectado gravemente a las empresas informativas $y$, si bien existen numerosas noticias que hablan del número de medios de comunicación cerrados o de periodistas despedidos, son mucho menos comunes los textos que hablan de las consecuencias sufridas en los centros documentales, quizá porque en los medios 
empiezan a percibirlos como obsoletos con la llegada de las nuevas tecnologías. Como bien explica Rubio-Lacoba (2010, p. 645):

"La crisis está acorralando a los servicios de documentación, que ven como sus plantillas se esquilman, sus recursos se congelan y su razón de ser es cuestionada (...) Digitalizando el archivo y contando con internet como oráculo documental, ¿para qué seguir gastando en un departamento cuyas tareas han sido absorbidas por informáticos y periodistas?".

Esta situación repercute directamente en la credibilidad y la calidad de los contenidos informativos ya que si el medio carece de departamento de documentación, o sus informaciones carecerán de datos que enriquezcan el futuro texto, o éstos no serán fiables. De cualquier manera, el producto final se verá afectado, de modo que será de menor calidad que la proporcionada por medios que sí posean centros de documentación, estos últimos, conseguirán a su vez niveles más altos de credibilidad (Codina, 2000, p. 49).

Ante la constatación de esta realidad, consideramos de interés revisar la situación actual de los servicios de documentación en prensa. Se juzgó más operativo ceñirnos a un espacio geográfico manejable y no cabe duda que el ámbito autonómico constituía un marco del máximo interés toda vez que, como afirman Ros-Martín y Rodero-Susiac (2009, p. 316), en España las empresas editoras de medios de comunicación regionales no han dedicado demasiados recursos a la gestión de la documentación tanto gráfica como textual. Así pues, se optó por actualizar el trabajo de Aquesolo-Vegas (1996) sobre los medios escritos en Andalucía, en el que se propuso estudiar la situación de los servicios de documentación de los principales periódicos andaluces en ese momento.

\section{Objetivos y método}

El presente trabajo tiene como objetivo conocer el estado actual de los centros documentales en los principales diarios andaluces, averiguando si cuentan con servicios de documentación o si fueron suprimidos desde el estudio anterior que nos sirve de referencia (Aquesolo-Vegas, 1996). Somos conscientes y queremos hacer explícita la limitación de este trabajo al comparar dos momentos separados dos décadas en el tiempo -con la enorme revolución que se ha experimentado en este terreno- pero que suponen la única renovación disponible de datos de este tipo, pues no contamos con estudios de estas características realizados en un periodo más reciente. En todo caso, creemos que los resultados arrojan luz sobre algunas cuestiones descriptivas que pueden ser de interés académico, docente y profesional al contar con datos actualizados que describen la realidad del presente partiendo de atrás, de donde venimos.

En concreto, entre los propósitos establecidos se incluye confirmar si el personal en los departamentos de documentación se ha visto disminuido, además de llevar a cabo una comprobación del progreso en la preparación de dicho personal. Asimismo, se pretende explorar la evolución de estos departamentos en cuanto al espacio físico que se les destina y, especialmente su situación en lo referente al equipamien- to con el que cuentan. Por último, pretendemos observar los cambios experimentados por estos centros con respecto a los servicios que ofrecen y la relación que mantienen con el equipo de redacción.

Dichos objetivos están guiados por algunas premisas de partida que intentamos corroborar, como la idea de que el departamento de documentación va a ser una de las parcelas perjudicadas en las empresas informativas, suprimiendo el servicio o mermando su personal y sus recursos en gran parte, al verse afectadas por recortes en sus presupuestos debido a la situación de crisis experimentada tanto en el sector de la prensa, como a nivel general desde aproximadamente 2008.

A la par -o incluso a pesar de- esta última proposición, nos planteamos a su vez constatar si, debido al arraigo de las TIC, los servicios han visto cómo sus infraestructuras experimentaban una mejora generalizada respecto a su equipamiento tecnológico.

El método de investigación seleccionado para el estudio del estado actual de los centros de documentación de los principales diarios andaluces ha sido la encuesta mediante cuestionario. Otros estudios precedentes -aunque no dedicados al estudio de los servicios documentales en prensa regional, cuya carencia queremos constatar a pesar del indudable interés que tendrían- han demostrado la eficacia del uso de encuestas a profesionales para obtener datos que nos ayuden en esta tarea descriptiva. Nos referimos por ejemplo a los ya citados de Micó-Sanz; Masip y García-Avilés (2009) y el estudio de Marcos-Recio y Edo (2015) sobre el uso de la documentación por parte de los periodistas españoles, así como la eficacia del funcionamiento de sus centros de documentación, realizado mediante encuesta aplicada a través de la Asociación de la Prensa de Madrid a un total de 246 profesionales; en aspectos más concretos, pero relacionados con el anterior estudio de carácter más integral, encontramos los trabajos de Guallar y Cornet (2004 y 2005) que cursaron encuestas, en este caso más similares a la de nuestro trabajo, a los departamentos de documentación de los diez diarios españoles de información general de más audiencia, tomando como referencia el Estudio general de medios (EGM), para indagar sobre la utilización de bases de datos comerciales y sobre la utilización de recursos web gratuitos, respectivamente; más recientemente, Orero y Cebrián-Enrique (2014) realizaron una encuesta a 81 documentalistas de medios de comunicación generalistas españoles sobre el uso de fuentes de información en centros de documentación, completada además mediante un estudio Delphi con ocho expertos en Documentación informativa; asimismo, en un campo más específico como el de la documentación fotográfica en prensa se han efectuado también investigaciones basadas en información suministrada por los responsables de dichos servicios (Sánchez-Vigil; MarcosRecio; Olivera-Zaldua, 2009 o, más recientemente, Guallar, 2011a).

Dado que nuestro propósito se basaba en actualizar los datos de la década de los 90, la base principal fue la encuesta de Aquesolo-Vegas (1996), combinada con la configuración de un corpus más acorde con los trabajos arriba referidos de 
Tabla 1. Datos de difusión diaria según $O J D$ de los principales diarios andaluces

\begin{tabular}{|l|c|}
\hline \multicolumn{1}{|c|}{ Periódico } & Difusión (enero de 2015) \\
\hline Ideal & 20.520 \\
\hline Sur & 17.547 \\
\hline Diario de Cádiz & 16.414 \\
\hline Diario de Sevilla & 15.241 \\
\hline Córdoba & 8.105 \\
\hline La voz de Almería & 5.211 \\
\hline Huelva información & 4.803 \\
\hline Jaén & 4.022 \\
\hline La opinión de Málaga & 2.690 \\
\hline Diario de Jerez & 4.465 \\
\hline Europa Sur & 2.366 \\
\hline Granada hoy & 2.320 \\
\hline Diario de Almería & 1.914 \\
\hline El día de Córdoba & 1.101 \\
\hline Total & 106.719 \\
\hline
\end{tabular}

Guallar y Cornet y enviada además a través de los medios online que actualmente están a nuestro alcance. Así pues, se diseñó y envió una encuesta con preguntas acerca del personal adscrito al servicio y su titulación, el horario y tipo de usuario, la ubicación del local y equipamiento, fondos disponibles o nivel de informatización y equipos y tipo de información que facilitan.

Frente al corpus tomado por Aquesolo que estaba compuesto en un primer momento por 11 diarios, -La voz de Almería, El diario de Cádiz, Córdoba, Ideal (Granada), Huelva información, Jaén, Sur (Málaga), Diario 16 Málaga, $A B C$ Sevilla, Diario 16 Andalucía (Sevilla) y El correo de Andalucía (Sevilla)-, de los que finalmente sólo pudo contar con la colaboración de siete, la presente investigación ha aumentado el número de rotativos a los que someter a examen. Debido a evidentes cambios en el panorama mediático andaluz no se pudo contar con las mismas cabeceras, pero se seleccionaron dos periódicos por provincia en función de sus datos de difusión (tabla 1).

Posteriormente, se tomaron algunas determinaciones para hacer el material más operativo: se incluyeron los títulos de Viva Jaén, Viva Huelva y La voz (Cádiz) al no haber segundos periódicos en la tabla para estas provincias. Se excluyó a Europa Sur y al Diario de Jerez, a pesar de ser importantes en difusión, porque elegimos solo capitales de provincia. Además, añadimos a $A B C$ Sevilla por la importancia de este periódico, pero finalmente no contestaron la encuesta, al igual que Diario Jaén, lo que redujo la muestra definitiva de 16 a 14 rotativos:

- Diario de Sevilla

http:// www.diariodesevilla.es

- Sur

http:// www.diariosur.es

- La opinión de Málaga

http://www.laopiniondemalaga.es
- Diario de Cádiz

http://www.diariodecadiz.es

- Lavoz

http://http://www.lavozdigital.es

- Ideal

http://www.ideal.es

- Granada hoy

http://www.granadahoy.com

- Diario Córdoba

http://www.diariocordoba.com

- El día de Córdoba

http://www.eldiadecordoba.es

- La voz de Almería

http://www.lavozdealmeria.es

- Diario de Almería

http://www.elalmeria.es

- Viva Jaén

http://andaluciainformacion.es/jaen

- Huelva información

http://www.huelvainformacion.es

- Viva Huelva

http://andaluciainformacion.es/huelv

A continuación, se procedió a localizar a los responsables de documentación de los diarios seleccionados. En este proceso aparecieron ciertos obstáculos: parte de los encuestados, debido a su limitado tiempo, no se ofrecía proclive a colaborar, pese a tratarse de preguntas muy breves y concretas expuestas en un sencillo cuestionario de Google Drive. En concreto, la herramienta adoptada a la hora de conocer la situación actual de estos departamentos consistió en un cuestionario idéntico diseñado expresamente para este trabajo, que utilizaba de base el empleado en el estudio de Aquesolo, pero adaptado al contexto actual que constaba de las siguientes preguntas:

- Personal adscrito al servicio y titulación: ¿Cuántas personas se encargan de este departamento? ¿Qué tipo de formación tiene el personal adscrito?

- Horario: ¿Qué horario tienen los trabajadores de este departamento?

- Ubicación e instalaciones: ¿Se encuentra el servicio de documentación próximo a la redacción? ¿Dónde está ubicado concretamente? ¿Qué tamaño posee?

- Equipamiento: ¿El equipamiento está informatizado? ¿Qué software emplea? ¿Sobre qué sistema operativo trabaja? ¿Qué hardware emplea? (describa sus características y número de aparatos) ¿Qué tipo de clasificación utiliza el departamento de documentación?

- Información que suministran: ¿Qué tipo de información es la más requerida?

Por último, recibidas las respuestas a lo largo del tercer trimestre de 2014, se extrajeron los resultados y se compararon los datos obtenidos en el trabajo publicado previamente por José Antonio Aquesolo con los derivados de nuestro análisis de las cabeceras andaluzas seleccionadas. 


\section{Resultados}

Uno de los grandes obstáculos en ambos estudios ha sido la comunicación con los diarios seleccionados en la muestra. Podemos decir que se repitieron los problemas expuestos en el estudio de Aquesolo, que no pudo contar con las respuestas de algunos de los periódicos escogidos. Tras intentar contactar en reiteradas ocasiones con los responsables de los departamentos seleccionados (en 1996 por teléfono y fax; en esta última muestra se intenta la comunicación mediante teléfono y correo electrónico), quedó constancia desde un inicio de que no todos los medios se mostrarían igual de accesibles. Así pues, de los 16 diarios a los que se intenta acceder en un principio, pese a los múltiples esfuerzos por lograr la comunicación, fue imposible contactar con el departamento de Documentación del Diario Jaén (Diario Jaén $S A$ ) y con el de $A B C$ Sevilla (Vocento) con el fin de que rellenasen el formulario. En el caso de Aquesolo, los periódicos que, a pesar de prometer una respuesta inmediata, no contestaron fueron Sur de Málaga, $A B C$ de Sevilla y los dos pertenecientes al Grupo 16.

Otra cuestión destacable es el hecho de que, aunque fueron 16 los diarios escogidos en un principio para el análisis, hay que tener en cuenta que son seis los grupos editoriales a los que pertenecen: El diario de Sevilla, el Diario de Cádiz, Granada hoy, El día de Córdoba, el Diario de Almería y Huelva información pertenecen al Grupo Joly. ABC de Sevilla, La voz, Sur e Ideal al Grupo Vocento. La Opinión de Málaga, a Prensa Ibérica. El Grupo Zeta posee el Diario Córdoba. Prisa controla La voz de Almería. Por último, Viva Jaén y Viva Huelva forman parte del grupo editorial Publicaciones del Sur SA. Es importante hacer alusión a esta situación, toda vez que uno de los primeros resultados es el hecho de que en algunos casos ha habido una centralización del departamento de documentación, como veremos más adelante.

El proceso de recogida de datos y la interpretación de las encuestas recibidas aporta detalles, quizá anecdóticos, pero muy significativos sobre la realidad de los servicios documentales en las empresas informativas. En una de las tomas de contacto se nos llegó a preguntar "qué entendíamos por un centro de documentación", por lo que no es raro que no tuvieran claro si disponían o no de tal servicio, cuando ni tan siquiera conocían las características de un departamento de este tipo. En otro caso, nos encontramos con que el diario Córdoba al rellenar el cuestionario afirma que posee un servicio de documentación pero a la par señala que no hay ninguna persona que se encargue de gestionarlo.

La información que los diarios suministraron en la encuesta en ocasiones fue incompleta o difusa. Como ejemplo, Ideal respondió a la pregunta de "qué horarios tienen los trabajadores de este departamento", indicando que la jornada laboral del responsable de este servicio suele ser de ocho horas, sin especificar una franja horaria aproximada. En todo caso, al igual que en el estudio de hace dos décadas se han procurado salvar estas lagunas y podemos decir que en la mayor parte de los casos, salvo las excepciones mencionadas, esta investigación no hubiera sido posible sin la colaboración de las fuentes implicadas, por lo que consideramos que debemos dejar constancia de nuestro agradecimiento.
Tabla 2. La tabla muestra, de entre los diarios analizados, cuáles tienen departamento de documentación (el símbolo " $\mathrm{X}$ " significa que el diario pertenece al Grupo Joly y que tiene este servicio centralizado en Sevilla)

\begin{tabular}{|l|c|c|c|}
\hline \multicolumn{1}{|c|}{ Cabecera } & $\begin{array}{c}\text { Tiene depto. de } \\
\text { documentación }\end{array}$ & No tiene & No contesta \\
\hline Diario de Sevilla & $\mathrm{X}$ & & \\
\hline Abc de Sevilla & $\mathrm{X}$ & $\mathrm{X}$ \\
\hline Sur & & $\mathrm{X}$ & \\
\hline La opinión de Málaga & $\mathrm{X}$ & & \\
\hline Diario de Cádiz & $\mathrm{X}$ & $\mathrm{X}$ & \\
\hline La voz & $\mathrm{X}$ & & \\
\hline Ideal & $\mathrm{X}$ & & \\
\hline Granada hoy & $\mathrm{X}$ & & \\
\hline Córdoba & $\mathrm{X}$ & & \\
\hline El día de Córdoba & $\mathrm{X}$ & & \\
\hline La voz de Almería & & $\mathrm{X}$ & \\
\hline Diario de Almería & & & \\
\hline Diario Jaén & & & \\
\hline Viva Jaén & & & \\
\hline Huelva información & & & \\
\hline Viva Huelva & & & \\
\hline
\end{tabular}

\subsection{Existencia de departamento de documentación}

En 1996, la investigación de Aquesolo reveló que todos los diarios, afirmaban tener "un archivo o centro de documentación que se pudiera identificar como tal", a excepción del Diario de Cádiz que tan sólo contaba con un archivo fotográfico. Casi todos los departamentos disponían de una persona que se encargase de la labor de documentalista, Ideal con dos (Aquesolo-Vegas, 1996).

En la actualidad no todos los diarios cuentan con un departamento de documentación. El diario La voz del grupo Vocento afirma contar tan sólo con una base de datos; Diario Córdoba, afirma que tiene un servicio de este tipo pero que no hay ninguna persona encargada de su gestión, es decir, cuenta más bien con un archivo digital que cualquier redactor puede gestionar desde su ordenador; Viva Jaén y Viva Huelva del grupo Publicaciones del Sur SA dejan esta labor en manos de los periodistas; en el caso de La opinión de Málaga, indican que fue suprimido. Entre los que sí tienen un departamento de documentación se encuentran los diarios del Grupo Joly: Diario de Sevilla, Diario de Cádiz, Granada hoy, Diario de Almería, El día de Córdoba y Huelva información, si bien comparten el mismo departamento centralizado en Sevilla del cual se encargan dos personas; La voz de Almería (Prisa) con una persona al cargo; y Sur e Ideal de Vocento tienen dos personas en el primero y una en el segundo que se encargan de la administración de dichos servicios. Los datos en lo referente a la cantidad de personal del departamento no salen bien parados de la comparación con los que arroja el estudio de Marcos-Recio y Edo (2015), que pidieron a los periodistas encuestados en su investigación que indicaran el número de documentalistas que trabajan en su medio: 
"un $14 \%$ respondió entre 5 y 10 documentalistas, un $17 \%$ entre 10 y 20 , un $18 \%$ más de 20 y, por último, un $48 \%$ entre 1 y 5 " (Marcos-Recio; Edo, 2015, p. 403).

A lo que dichos autores añaden:

"según estas respuestas ha bajado el número con respecto a hace una década, donde la mayoría de los centros contaban con más de 20 documentalistas. Se considera que la crisis que atraviesan los medios también se ha reflejado en los centros de documentación, ya que han perdido documentalistas cuando el medio ha despedido a parte de su plantilla" (Marcos-Recio; Edo, 2015, p. 403).

\subsection{Personal adscrito al servicio y titulación}

Dentro de los trabajadores de este departamento, en lo que se refiere a la titulación, en 1996, dos eran procedentes de formación profesional, otros dos de estudios de bachillerato superior, uno poseía un título de grado medio (sin especificar), uno era redactor gráfico y dos no contestaron. Es decir, no se encontraba ningún documentalista titulado, bien en ciencias de la información o de la comunicación, bien en biblioteconomía y documentación, que son las titulaciones más afines.

Respecto a la titulación actual de dicho personal, en el caso del Grupo Joly poseen una licenciatura y cursos en formación de biblioteconomía y documentación; en $L a$ Voz de Almería la formación se queda en la de estudios de "administración", según se expresó en la encuesta; licenciados en periodismo en el diario Sur y, en Ideal, una licenciada en ciencias de la información con un máster en documentación digital, con lo que se advierte una notable mejora en la formación de los profesionales. La figura 1 muestra cómo en 1996 el 75\% del personal que se encontraba a cargo de este servicio provenía de estudios de grado medio, mientras que en la figura 2 se advierte como ese porcentaje baja hasta el $25 \%$ y nos encontramos con que el $75 \%$ del personal posee estudios de grado superior.

\subsection{Horario}

En el estudio de 1996 la mayoría de los diarios afirmaban que la tarde era el período más importante -las horas iban entre las $16: 00$ y las 24:00-. En el caso de El correo de An-

\section{Nivel de estudios en 1996}

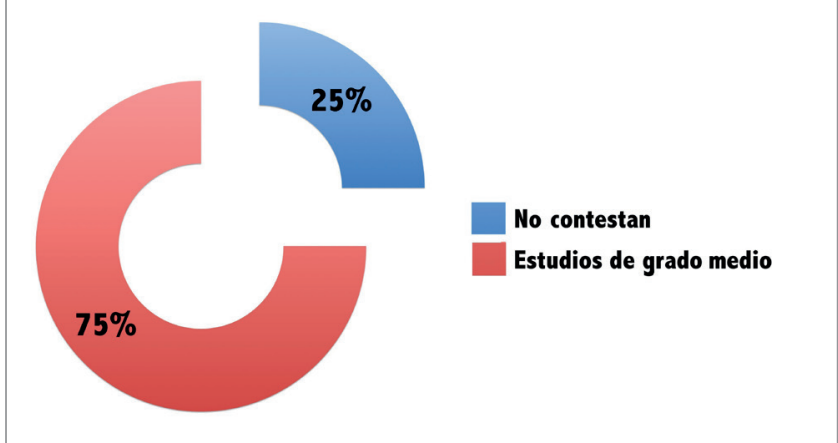

Figura 1. Personal con estudios de grado medio en las cabeceras andaluzas en 1996. Fuente: Aquesolo (1996). dalucía y Huelva información abrían el servicio por las mañanas. Córdoba y La voz de Almería no respondieron a esta pregunta en concreto (Aquesolo-Vegas, 1996).

En nuestro estudio el horario varía bastante de un diario a otro: desde la existencia de dos turnos divididos entre las 9:30h y las 21:00h en el Grupo Joly, o el caso de Sur que también se divide entre la mañana y la tarde, al de $L a$ voz de Almería que tan sólo cuenta con turno de mañana (de 9:00h a 14:00h); Ideal no especifica el horario pero afirma que suele ser de 8 horas (no indica la franja horaria) y añade a su vez que "depende del día".

\subsection{Ubicación e instalaciones}

En la década de los 90 los locales habilitados con el fin de ser utilizados como servicios de documentación se hallaban próximos a la redacción (incluso formaban parte de ésta). Con la excepción de Huelva información que lo situaba en otra planta, junto a la sala de administración. El espacio asignado variaba bastante de unos diarios a otros, yendo desde los $4 \mathrm{~m}^{2}$ de Huelva información, a los $14 \mathrm{~m}^{2}$ de Córdoba, pasando por los $35 \mathrm{~m}^{2}$ de El correo de Andalucía y los $50 \mathrm{~m}^{2}$ de Diario de Cádiz, hasta alcanzar los $95 \mathrm{~m}^{2}$ de Ideal (Aquesolo-Vegas, 1996).

En nuestros días la situación también es bastante diversa dependiendo de la cabecera a examinar. Los espacios habilitados con este fin están situados cerca de la redacción o forman parte de la misma. Su tamaño varía de las "dos mesas" en el diario Ideal, a los $2 \mathrm{~m}^{2}$ de La voz de Almería, pasando por los $12 \mathrm{~m}^{2}$ del diario Sur, hasta llegar a los aproximadamente $25 \mathrm{~m}^{2}$ del Grupo Joly.

\subsection{Equipamiento}

En los resultados obtenidos por Aquesolo, El correo de Andalucía era el único que tenía parte de su archivo informatizado (la recepción, búsqueda y archivo de fotografías). En los demás diarios ninguno contaba con archivos informatizados; su sistema de archivos, en general, se basaba en carpetas en las cuales los recortes se clasificaban y almacenaban por temas y estructuras temáticas. Córdoba, El correo de Andalucía y Jaén disponían de disco óptico para almacenar las imágenes. El resto del equipamiento se limitaba a alguna fotocopiadora o fax (Aquesolo-Vegas, 1996).

\section{Nivel de estudios en 2014}

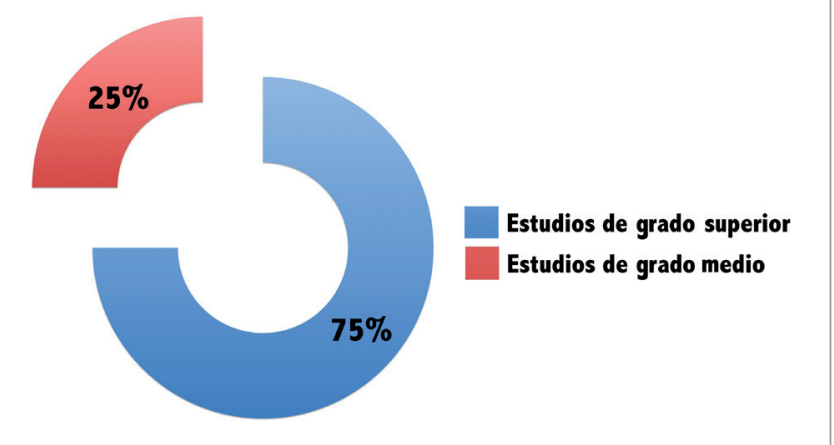

Figura 2. Personal con estudios de grado medio y de grado superior en las cabeceras andaluzas 
Mientras, nuestro análisis muestra cómo en estos momentos todos poseen un equipamiento informático suficiente: en lo que se refiere al hardware, el Grupo Joly habla de dos ordenadores, Córdoba entre sus equipos cuenta con un servidor HP Proliant DL360 y La Voz de Almería confirma que la información puede ser consultada desde "cualquier ordenador de redacción". Sin embargo, Sur e Ideal directamente no responden a esta pregunta del cuestionario. Respecto al software de dichos departamentos, el Grupo Joly afirmaba que es "una producción propia del departamento de informática"; La voz de Almería indicó que dispone de "un buscador creado por la propia empresa"; en el diario Córdoba el software fue "realizado por el departamento de I+D de Grupo Zeta", al cual pertenece dicha cabecera; Sur se limitaba a confirmar que era "propio del periódico"; y, por último, Ideal nos refiere a "Aplidoc, elaborado para los periódicos pertenecientes al Grupo Vocento".

\subsection{Información que suministran}

En el estudio de la década de los 90 los periódicos afirmaban que su fondo de archivo estaba compuesto fundamentalmente por revistas a las que estaban abonados, boletines oficiales e información proveniente de publicaciones anteriores del propio diario (Aquesolo-Vegas, 1996). Estos contenidos, como vemos, fundamentalmente en papel, eran los que entonces los departamentos podían proporcionar para completar las informaciones.

Cuando preguntamos a los responsables del departamento de documentación de cada diario cuál era la información más requerida, el Grupo Joly manifestó que en su caso se trataba de "noticias anteriores del caso que se investiga, publicadas por su propio medio", además de "fotografías de archivo"; Sur afirmó que tomaban como base "la información publicada en el periódico" y la complementaban "con información directa proporcionada por los redactores". La voz de Almería simplemente se limitaba a responder que "de todo tipo"; el diario Córdoba contestaba vagamente que las más solicitadas eran las "informaciones publicadas de temas de actualidad"; e Ideal declara que su mayor demanda es la de información "local de Granada, Jaén y Almería (datos estadísticos, dossiers históricos sobre temas locales, biografías sobre personajes locales)". Estos tres últimos no especificaban si la información era propia o proveniente de otras fuentes.

De lo anterior se deduce que, de todos los datos que suministran los departamentos en la actualidad, los más requeridos son noticias y fotos de archivo del propio periódico, en su mayoría de informaciones locales que puedan ser demandadas por los redactores con posterioridad. Puede decirse que, aunque con soportes diferentes, las demandas son parecidas y derivan también del tipo de periódicos que estamos estudiando, apegados como es lógico a las informaciones territoriales de más corto alcance.

\section{Discusión y conclusiones}

Observando los resultados obtenidos, coincidimos con la apreciación de Javier Guallar cuando afirma que, pese a la importancia del trabajo documental, éste está escasamente valorado, sobre todo cuanto más pequeña es la empresa informativa. Según Guallar (2011b, pp. 65-66), son numerosas las redacciones que cuentan con un departamento de documentación y con profesionales de esta especialidad. En general, las empresas de tamaño medio o grande cuentan con un centro de este tipo, siendo las más pequeñas las que tienen dificultades para mantener estos servicios en funcionamiento y actualizados.

Aunque después de comparar los datos obtenidos en ambos estudios se pueda llegar a la rápida e incompleta conclusión -fruto quizá de las limitaciones más arriba comentadas- de que, en proporción, hay el mismo número de periódicos que cuentan con un servicio de documentación que en 1996, la realidad es más complicada: si bien podría afirmarse lo anterior, al realizar un examen más detallado se percibe que el número de departamentos se ha visto reducido al centralizar sus servicios entre los mismos periódicos de un grupo editorial.

Para soportar los costes se tiende a una fuerte concentración mediática a fin de compartir recursos y abaratar gastos. Se generan

"fuertes procesos de reconversión y la afloración de intensivas dinámicas de innovación" (Campos-Freire, 2010, p. 14).

Todo esto se traduce en que, por ejemplo, un grupo mediático como Joly haya centralizado en Sevilla el departamento de documentación para todos sus diarios dentro de la Comunidad Andaluza, tanto en papel como en digital.

Hecha esta primera constatación, con todo, tras analizar los resultados obtenidos y compararlos con los que cosechó José-Antonio Aquesolo-Vegas en 1996, podemos afirmar que se observan aspectos tanto positivos como negativos.

Entre los positivos destaca sin duda la mejora en la preparación del personal adscrito a estos departamentos: el estudio de Aquesolo apuntaba cómo el perfil profesional del personal no era acorde al trabajo a desempeñar -graduados en enseñanzas de grado medio- sin embargo, los datos obtenidos en las encuestas suministradas a los periódicos en esta última muestra perfilan una notable mejora. Lo general es que el personal cuente con un título de enseñanza superior relacionado directamente con la documentación (a excepción de La voz de Almería cuyo responsable está formado en enseñanzas administrativas).

Además, hoy podemos resaltar el avance en la informatización del equipamiento, que en 1996 era prácticamente inexistente y ahora es un hecho: toda la información se encuentra automatizada y plenamente interconectada de forma que los redactores pueden acceder a ésta cuando es precisa su recuperación para la contextualización de una noticia. Según evidencian los datos obtenidos, el avance en los equipos ha experimentado un impulso abrumador. El estudio de Aquesolo reflejaba que eran muy pocos los periódicos que tenían automatizado su centro de documentación, salvo excepciones como el Correo de Andalucía que tenía informatizada la recepción, búsqueda y archivo de material fotográfico y cuyos servicios ya permitían la conexión en red y la accesibilidad a la información almacenada. No obstante, en dicho estudio ya se presentía su pronta implantación: 
los que afirmaban carecer de informatización en el servicio señalaban su intención de incorporarla.

Los datos obtenidos casi dos décadas más tarde son una clara manifestación del arraigo de las TIC en nuestra sociedad, pues no hay un sólo diario, entre aquellos que cuentan con un servicio de este tipo, cuyo equipamiento no se halle informatizado y en el cual la conexión en red y la accesibilidad no sean una realidad; es más, lo habitual es que cada grupo editorial cuente con un software de creación propia que rija su sistema informático.

Ambos resultados, sobre personal cualificado y equipamiento actualizado, resultan contradictorios con respecto a la casi extinción de los departamentos de documentación, aunque se explican sobre todo por la gran tendencia a la centralización que se deriva de la concentración de las empresas editoriales. No obstante, no basta con dotar un espacio del equipamiento y personal adecuado para conseguir un servicio de documentación satisfactorio: hace falta un esfuerzo motivador para alcanzar una cultura organizacional orientada a compartir y generar conocimientos, mediante el aprovechamiento de las capacidades creativas e innovadoras del factor humano. Los trabajadores originan el capital intelectual, por lo que sería saludable que la empresa potenciase las diferencias intelectuales, facilitando la generación de ideas y su puesta en práctica (Reyes-Ramírez, 2007).

Debemos efectuar un último apunte antes de cerrar esta cuestión llamando la atención ante el hecho de que, pese a que el volumen de información que tiene que gestionar los departamentos de documentación aumenta cada día de manera ingente, éstos cuentan en la actualidad con aproximadamente el mismo número de personas para su administración que hace dos décadas cuando Aquesolo realizó su estudio.

Entramos así en los aspectos negativos que se derivan de los resultados de este trabajo: se observa como en 1996 los diarios implementaban la funcionalidad de sus departamentos documentales, en una clara etapa de despunte tecnológico lo cual suponía introducir mejoras en un servicio insustituible. Sin embargo, en el momento actual, algunos de los diarios analizados perciben una decadencia en su razón de ser y los consideran prescindibles -como ocurre con La opinión de Málaga donde, en determinados momentos como el actual, se prescinde de este departamento- e, incluso, obsoletos -en el caso de Viva Huelva y Viva Jaén la persona responsable de atender la llamada de contacto confiesa que esa labor recae sobre los propios periodistas-ya que se considera innecesario e, incluso, para algunos resulta impensable realizar una inversión dirigida a impulsar estos servicios.

Con todo, los periodistas aseguran no tener dudas de la importancia que posee la documentación como parte de un adecuado proceso informativo. Resulta irónico, si se tiene en cuenta que son contadas las cabeceras españolas que cuentan con un equipo de investigación, hecho que se achaca a la idea de que todo periodista realiza una labor de investigación innata a su labor profesional y al elevado coste que supone el mantenimiento de un equipo de este tipo (Díaz-Güell, 2003, pp. 58-59). Pero quizá porque piensen que el departamento de documentación es tan sólo un es- pacio físico, y desconocen el perfil y aptitudes del documentalista, viéndolos como meros organizadores del archivo, como si se encargaran en exclusiva del trabajo que ejecuta un bibliotecario. En este sentido, el $50 \%$ de los periodistas encuestados en el estudio de 2009 referido con anterioridad apostaban por la futura desaparición de los departamentos de documentación al ser suplidos por internet (Micó-Sanz; Masip; García-Avilés, 2009, p. 288).

Y sin embargo, curiosamente, el centro de documentación no tendría por qué ser un desconocido para los redactores, teniendo en cuenta su integración y su proximidad física. La cercanía de la sala de redacción respecto a la del departamento de documentación es la opción habitual, aunque tampoco se ve como imprescindible actualmente debido a la tecnología empleada. Cualquier redactor puede acceder al fondo documental sin tener que desplazarse de su espacio de trabajo, basta con un clic en el teclado. Así pues, resulta interesante destacar que, aunque tanto en el estudio de 1996 como en el actual, los departamentos de documentación cuentan con un tamaño que varía drásticamente de unos a otros, rozando en algunos casos lo incomprensible (por ejemplo, las "dos mesas" disponibles para esa tarea en el diario Ideal o los dos metros cuadrados que le reserva La voz de Almería), en general, los dimensiones que se dedicaban a estos espacios se han visto reducidas: el caso más llamativo lo encontramos al analizar el servicio de documentación del diario ldeal que antes contaba con $95 \mathrm{~m}^{2}$. Lo expuesto no es de extrañar si se tiene en cuenta que se ha pasado de un sistema de almacenamiento físico a otro digital, pero también nos aporta un dato simbólico respecto a la consideración de estos espacios.

Hay que reseñar además que la crisis económica sumada a la, digamos, identitaria de la prensa también ha hecho mella, como se pudo apreciar en la conversación telefónica mantenida con una trabajadora de La opinión de Málaga que confirmaba que esa era una de las principales razones por las que se había suprimido el departamento de documentación de dicho diario.

Así pues, ante este escenario, queda en entredicho la figura del documentalista informativo tal y como hasta ahora estaba planteada y surgen nuevas propuestas:

"En el centro de documentación hemos pasado de un simple diálogo -y en ocasiones monólogo- a una conversación permanente en la que se han de facilitar respuestas rápidas y precisas sobre las necesidades informativas, no sólo de los propios periodistas, sino de aquellos usuarios que estén dispuestos a pagar por ella" (Marcos-Recio, 1998, p. 124).

Siguiendo a Guallar, sin embargo, no todos los profesionales parecen entender por igual la importancia de estos nuevos productos y servicios que, pese a no ser plenamente explotados, son su mejor arma. Los documentalistas y los centros de documentación deben encaminarse a la creación de contenidos, superando en la medida de lo posible anteriores roles subordinados y de intermediación (Guallar, 2011b, p. 66).

Así, entre las nuevas funciones que se vienen proponiendo, destaca también la de la gestión y difusión de recursos disponibles en el ciberespacio dentro de la propia empresa, 
que ya se empezaba a subrayar en estudios de hace dos décadas como citábamos más arriba:

"Con expertos documentalistas en el manejo de estas redes, para hacer una acertada selección, de tal forma que sirvan como filtro de documentos que han de entregar al periodista, quien ha de asumir por su parte la confianza plena de que esa selección se ha llevado a cabo con criterio y rigor periodístico y documental, lo que no incidirá de forma negativa en la elaboración final de la información" (Marcos-Recio, 1998, p. 118).

Posteriormente se ha continuado defendiendo la idea de que el documentalista puede ser hoy un orientador que ayude a seleccionar las mejores fuentes (García-González, 2006, p. 6).

Pese a los limitados conocimientos y a la falta de formación que los periodistas admiten tener de la búsqueda de información en la Red, dichos profesionales parecen preferirla frente a sus propias bases de datos. Cabe recordar que son minoría los diarios que ofrecieron cursos de formación a sus trabajadores sobre un uso adecuado de internet, hecho que repercute negativamente en la información ofrecida por los periodistas y que se refleja en un descenso en la calidad de la información (Micó-Sanz; Masip; García-Avilés, 2009, p. 287).

Sin embargo, hoy comprobamos cómo estas propuestas, aun siguiendo vigentes, no se han secundado en la práctica. Con todo, esperamos que estudios como éste que llamen la atención sobre la situación actual de los servicios de documentación en la prensa, contribuyan a una reflexión pues coincidimos en que los documentalistas informativos pueden convertirse en el remo del que se vale el periodista para navegar en el mar de datos que ha provocado el acceso masivo a la Web y la consiguiente saturación informativa, pues los documentalistas

"aportan una perspectiva totalmente diferente a las redacciones" al centrarse "en el pasado y el futuro" (Paul, 2009).

\section{Bibliografía}

Aquesolo-Vegas, José-Antonio (1996). "Situación de los servicios de documentación de la prensa diaria de Andalucía". Cuadernos de documentación multimedia, n. 5, pp. 69-73. http://pendientedemigracion.ucm.es/info/multidoc/ multidoc/revista/cuadern5/aquesolo.htm

Campos-Freire, Francisco (2010). "Los nuevos modelos de gestión de las empresas mediáticas". Estudios sobre el mensaje periodístico, n. 16, p. 13-30.

http://revistas.ucm.es/index.php/ESMP/article/view/ ESMP1010110013A

Codina, Lluís (2000). "La documentación de los medios de comunicación: Situación actual y perspectivas de futuro", Cuadernos de documentación multimedia, n. 10, pp. 49-66. https://dialnet.unirioja.es/servlet/articulo?codigo=1408512

Díaz-Güell, Luis (2003). Periodismo y periodistas de investigación en España, 1975-2000: contribución al cambio político, jurídico, económico y social. Tesis doctoral. Universidad
Complutense de Madrid, Departamento de Periodismo III. http://eprints.ucm.es/4843

Estrada-Nora-González, Belén; Patallo-Fernández, Cristina; Pastor-Blanco, Marcela (2009). "Puesta en marcha de un servicio de documentación digital en la Televisión del Principado de Asturias (TPA)". El profesional de la información, v. 18 , n. 3, pp. 326-332.

http://dx.doi.org/10.3145/epi.2009.may.11

Galdón-López, Gabriel (2002). Teoría y práctica de la documentación informativa. Barcelona: Ariel. ISBN: 978 8434412934

González-Quesada, Alfonso (2003). "La investigación en documentación informativa en España”. Documentación de las ciencias de la información, v. 26, pp. 71-97.

http://revistas.ucm.es/index.php/DCIN/article/view/ DCIN0303110071A

Guallar, Javier (2011a). “Documentación fotográfica en la prensa. Casos de El país, El periódico y La vanguardia". El profesional de la información, v. 20, n. 4, pp. 392-398. http://dx.doi.org/10.3145/epi.2011.jul.05

Guallar, Javier (2011b). "La documentación en la prensa digital. Nuevas tendencias y perspectivas". En: III Congreso internacional de ciberperiodismo y web 2.0. La transformación del espacio mediático, pp.52-68.

http://eprints.rclis.org/16326

Guallar, Javier; Cornet, Anna (2004). "Fuentes de información digitales en los centros de documentación de prensa: las bases de datos comerciales". El profesional de la información, v. 13, n. 2, pp. 107-117.

http://www.elprofesionaldelainformacion.com/contenidos/2004/ marzo/4.pdf

Guallar, Javier; Cornet, Anna (2005). "Fuentes de información digitales en los centros de documentación de prensa: recursos web gratuitos". El profesional de la información, v. 14, n. 4, p. 296-308.

http://www.elprofesionaldelainformacion.com/contenidos/2005/ julio/9.pdf

Marcos-Recio, Juan-Carlos (1998). "Una nueva concepción de la documentación en los medios electrónicos: Retos y nuevas tareas profesionales". Documentación de las ciencias de la información, n. 21, pp. 113-130.

http://eprints.ucm.es/7023

Marcos-Recio, Juan-Carlos; Edo, Concha (2015). “Análisis de la nueva perspectiva de la documentación periodística en los medios de comunicación españoles". Revista general de información y documentación, v. 25, n. 2, pp. 389-423. http://dx.doi.org/10.5209/rev_RGID.2015.v25.n2.51235

Marcos-Recio, Juan-Carlos; Sánchez-Vigil, Juan-Miguel; Olivera-Zaldua, María (2010). "Modelos de gestión documental en las agencias de publicidad". El profesional de la información, v. 19, n. 2, pp. 175-183.

http://dx.doi.org/10.3145/epi.2010.mar.08

Micó-Sanz, Josep-Lluís; Masip, Pere; García-Avilés, JoséAlberto (2009). "Periodistas que ejercen de documentalistas (¿y viceversa?). Nuevas relaciones entre la redacción y el 
archivo tras la digitalización de los medios". El profesional de la información, v. 18, n. 3, pp. 284-290.

http://dx.doi.org/10.3145/epi.2009.may.05

Moreiro-González, José-Antonio (coord.) (2000). Manual de documentación informativa. Madrid: Cátedra. ISBN: 978 8437617985

Orero, Paz; Cebrián-Enrique, Bernardino J. (2014). "Criterios de evaluación y aplicación de fuentes de información web en centros de documentación periodística". El profesional de la información, v. 23, n. 6, pp. 612-617.

http://dx.doi.org/10.3145/epi.2014.nov.08

Paul, Nora (2009). “Elegía del centro de documentación de prensa", El profesional de la información, v. 18, n.3, pp. 249-253.

http://dx.doi.org/10.3145/epi.2009.may.01

Reyes-Ramírez, Livia-Mercedes (2007). "Sistemas de información para la prensa: la gestión de la información y el conocimiento en el contexto de los sistemas integrados de información". Acimed, v. 15, n. 2.

http://eprints.rclis.org/9104/1/Sistemas_de_informaci\%C3\%B3n para_la_prensa.pdf

Ros-Martín, Marcos; Rodero-Susiac, Alfonso (2009). "El servicio de documentación de un medio impreso regional: evolución en el diario Levante-EMV". El profesional de la información, v. 18, n. 3, pp. 316-322.

http://dx.doi.org/10.3145/epi.2009.may.09

Rubio-Lacoba, María (2005). "Nuevos tiempos para la documentación informativa en el periodismo digital: viejas y nuevas funciones del servicio de documentación digital". Comunicación y sociedad, v. 18, n. 1, pp. 153-168.

http://eprints.rclis.org/16327

Rubio-Lacoba, María (2010). "Documentalistas de prensa, ¿cuál es vuestro oficio?”. El profesional de la información, v. 19, n. 6, pp. 645-651.

http://dx.doi.org/10.3145/epi.2010.nov.11

Sánchez-Vigil, Juan-Miguel; Marcos-Recio, Juan-Carlos; Olivera-Zaldua, María (2009). "Influencia de la fotografía digital en los departamentos de documentación de prensa". El profesional de la información, v. 18, n. 3, pp. 278-283.

http://dx.doi.org/10.3145/epi.2009.may.04

Torres-Rodríguez, Vanessa-Berenice (2012). Documentación periodística. México: Red Tercer Milenio. ISBN: 978607 7330424

\title{
Colección EPI Scholar
}

\section{Libros científicos de Información, Documentación y Comunicación}

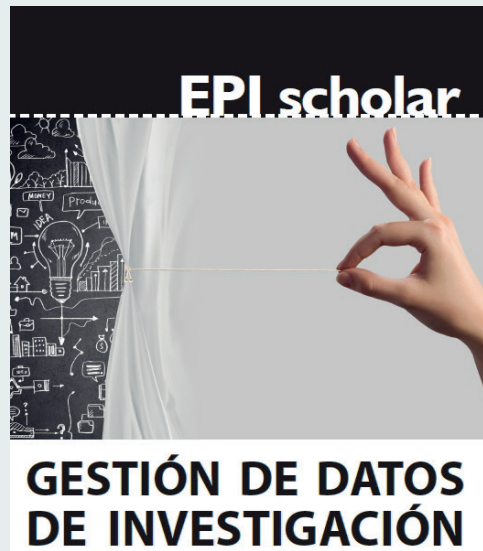

\section{FABIANO COUTO CORRÉA}

\section{Gestión de datos de investigación de Fabiano Couto Corrêa}

Vivimos momentos de auténtica expectación por el papel que el acceso a grandes cantidades de datos de investigaciones puede llegar a jugar en el presente y el futuro inmediato en el desarrollo del conocimiento en todas las disciplinas. Para ello es absolutamente clave la existencia de procedimientos adecuados en la gestión de los datos de investigación.

En este libro se presentan diversas recomendaciones para ello. Las recomendaciones están dirigidas en primer lugar a investigadores. $Y$ como los bibliotecarios tienen un papel cada vez más importante en estos procesos, se propone también un enfoque adicional: "ayudarles a ayudar a los investigadores". Así, ambos colectivos pueden encontrar en estas páginas una guía tremendamente útil para la gestión de los datos de investigación.

Del prefacio de Fernanda Peset: "Aunque gestionar el conocimiento en el ámbito de las ciencias es extremadamente complejo, en este momento enfrentamos la oportunidad de convertir en realidad el acceso a los resultados de la investigación en su más amplia acepción. Y el libro que tienen en las manos, les ayudará a comprender e involucrarse en las diferentes dimensiones de la gestión de los datos de investigación".

Couto Corrêa, Fabiano (2016). Gestión de datos de investigación. Barcelona: El profesional de la información, Editorial UOC, colección EPI Scholar n. 6, 164 pp. ISBN: 9788491164302

\author{
Información: Isabel Olea \\ epi.iolea@gmail.com \\ http://www.elprofesionaldelainformacion.com/librosEPIScholar.html
}

